hep-th/0508097

\title{
Linear Sigma Models of H and KK Monopoles
}

\author{
Kazumi Okuyama \\ Department of Physics and Astronomy, University of British Columbia \\ Vancouver, BC, V6T 1Z1, Canada \\ kazumi@phas.ubc.ca
}

\begin{abstract}
We propose a gauged linear sigma model of $k \mathrm{H}$-monopoles. We also consider the T-dual of this model describing KK-monopoles and clarify the meaning of "winding coordinate" studied recently in hep-th/0507204.
\end{abstract}

August 2005 


\section{Introduction}

The T-duality between H-monopoles (NS5-branes on $\mathbf{S}^{1}$ ) [1, 2, 3] and KK-monopoles (multi-Taub-NUT space) [4.5] is a well-established duality [6]. At the level of supergravity this duality exchanges the two gauge fields $B_{i 9}$ and $g_{i 9}$ where $i=6,7,8$ and $x^{9}$ is the $\mathbf{S}^{1}$ direction (we take the worldvolume of monopoles to be extended in the 012345 directions).

However, it was pointed out [7] that to fully understand the correspondence of collective coordinates of these monopoles we have to take into account of the stringy effects. A single H-monopole carries collective coordinates $\mathbf{R}^{3} \times \mathbf{S}^{1}$ which correspond to the transverse space of the NS5-brane $\mathbf{E}$. On the other hand, the Taub-NUT space has a moduli $\mathbf{R}^{3}$ which specifies the degeneration locus of the $\mathbf{S}^{1}$ fiber and hence $\mathbf{S}^{1}$ part of the moduli on the $\mathrm{H}$-monopole side is missing in the geometric picture of KK-monopoles. In other words, KK-monopoles are smeared in the $\mathbf{S}^{1}$ direction and there is no definite position in $\mathbf{S}^{1}$. It is known that the missing moduli comes from the zero-modes of the B-field given by the harmonic two-forms on the Taub-NUT space [10]. Since B-field couples to the winding number, the $\mathbf{S}^{1}$ moduli of KK-monopole is not a geometric translation mode but the shift symmetry in the "winding coordinate" [7].

In a recent paper [11], it is argued that a gauged linear sigma model (GLSM) [12] is a useful tool to analyze this problem. In [11] the GLSM of a single KK-monopole was studied by taking the T-dual of the GLSM for a H-monopole obtained by Tong [13], and the worldsheet instanton corrections to the effective sigma-model metric is computed. This result seems puzzling from the effective nonlinear sigma model perspective since there is no closed two-cycle in a single KK-monopole background where the worldsheet is supposed to wrap.

In this paper, we generalize [13,11] and construct the GLSM of multiple H-monopoles and its T-dual GLSM of the multiple KK-monopoles. We argue that the moduli of $\mathrm{H}$ - and KK-monopoles appear in GLSM as parameters of two-dimensional field theory and clarify the physical meaning of "winding moduli" of KK-monopoles by analyzing the effective theory obtained from the GLSM. We also suggest a possible resolution to the abovementioned puzzle.

The organization of this paper is as follows. In section 2, we construct a GLSM for $k$ $\mathrm{H}$-monopoles and study its low energy behavior and instanton corrections. In section 3, by

1 We consider NS5-branes in Type IIB theory. NS5-branes in Type IIA theory have extra $\mathbf{S}^{1}$ moduli [8,9]. 
taking the T-duality of H-monopole GLSM we obtain the GLSM for $k$ KK-monopoles. In section 4, we discuss the instanton effect of KK-monopole model from the effective theory perspective.

\section{GLSM for H-monopoles}

In this section, we construct a GLSM for the $k$ H-monopoles by generalizing the known model for $k=1$ [13]. Our model is a $\mathcal{N}=(4,4) U(1)^{k}$ gauge theory with $k$ charged hypermultiplets and one neutral twisted hypermultiplet.

\subsection{The Model}

We use the $\mathcal{N}=2$ superfield notation. We argue that $k \mathrm{H}$-monopoles are described by the GLSM with the following $D$-term $\mathcal{L}_{D}$, the superpotential term $\mathcal{L}_{F}$, and the twisted superpotential term $\mathcal{L}_{\widetilde{F}}$ :

$$
\begin{aligned}
& \mathcal{L}_{D}=\int d^{4} \theta \frac{1}{g^{2}}\left(-\Theta^{\dagger} \Theta+\Psi^{\dagger} \Psi\right)+\sum_{a=1}^{k}\left\{\frac{1}{e_{a}^{2}}\left(-\Sigma_{a}^{\dagger} \Sigma_{a}+\Phi_{a}^{\dagger} \Phi_{a}\right)+Q_{a}^{\dagger} e^{V_{a}} Q_{a}+\widetilde{Q}_{a}^{\dagger} e^{-V_{a}} \widetilde{Q}_{a}\right\} \\
& \mathcal{L}_{F}=\int d \theta^{+} d \theta^{-} \sum_{a=1}^{k}\left\{\widetilde{Q}_{a} \Phi_{a} Q_{a}+\left(s_{a}-\Psi\right) \Phi_{a}\right\}, \quad \mathcal{L}_{\widetilde{F}}=\int d \theta^{+} d \bar{\theta}^{-} \sum_{a=1}^{k}\left(t_{a}-\Theta\right) \Sigma_{a}
\end{aligned}
$$

Let us explain our notation in $(2.1) .\left(\Phi_{a}, \Sigma_{a}\right)$ is the $\mathcal{N}=4 U(1)$ vector multiplet, $\left(Q_{a}, \widetilde{Q}_{a}\right)$ is the charged hypermultiplet, and $(\Psi, \Theta)$ is the neutral twisted hypermultiplet. We can see that (2.1) reduces to the model in [13] when we set $k=1$. $\left(s_{a}, t_{a}\right)$ in $\mathcal{L}_{F}$ and $\mathcal{L}_{\widetilde{F}}$ are some constant parameters corresponding to the FI-parameters and the theta-angle

$$
s_{a}=r_{a}^{1}+i r_{a}^{2}, \quad t_{a}=r_{a}^{3}+i \theta_{a}
$$

In the following, we use the lower case letters for the scalar field component of the superfield except for the twisted hypermultiplet. Following [13], we denote the scalar component of twisted hypermultiplet as $\left(r^{1}, r^{2}, r^{3}, \theta\right)$ :

$$
\begin{aligned}
& \Psi=r^{1}+i r^{2}+\sqrt{2} \theta^{+} \chi_{+}+\sqrt{2} \theta^{-} \chi_{-}+\cdots \\
& \Theta=r^{3}+i \theta-i \sqrt{2} \theta^{+} \overline{\widetilde{\chi}}_{+}-i \sqrt{2} \bar{\theta}^{-} \widetilde{\chi}_{-}+\cdots
\end{aligned}
$$

We also use the vector notation $\vec{r}=\left(r^{1}, r^{2}, r^{3}\right)$ to emphasize the triplet of R-symmetry $S U(2)_{R}$. The important property of this Lagrangian is that the scalar fields $(\vec{r}, \theta)$ act as the dynamical FI-parameters and the dynamical theta-angle. 
It is easy to write down the component field expression of the Lagrangian in the Wess-Zumino gauge. Here we focus on the bosonic part of the action

$$
S=\frac{1}{2 \pi} \int d^{2} x\left(\mathcal{L}_{\text {kin }}+\mathcal{L}_{\text {pot }}+\mathcal{L}_{\text {top }}+\mathcal{L}_{\text {fermion }}\right)
$$

where $\mathcal{L}_{\text {kin }}$ is the kinetic term of bosonic fields

$$
\mathcal{L}_{\text {kin }}=-\frac{1}{2 g^{2}}\left((\partial \vec{r})^{2}+(\partial \theta)^{2}\right)+\sum_{a=1}^{k}\left\{\frac{1}{2 e_{a}^{2}}\left(\left(F_{01}^{a}\right)^{2}-\left|\partial \phi_{a}\right|^{2}-\left|\partial \sigma_{a}\right|^{2}\right)-\left|\mathcal{D} q_{a}\right|^{2}-\left|\mathcal{D} \widetilde{q}_{a}\right|^{2}\right\}
$$

and $\mathcal{L}_{\text {pot }}$ is the potential term

$$
\begin{aligned}
\mathcal{L}_{\text {pot }}=\sum_{a=1}^{k}\{ & -\frac{e_{a}^{2}}{2}\left(\left|q_{a}\right|^{2}-\left|\widetilde{q}_{a}\right|^{2}-r^{3}+r_{a}^{3}\right)^{2}-\frac{e_{a}^{2}}{2}\left|2 q_{a} \widetilde{q}_{a}-\left(r^{1}+i r^{2}\right)+\left(r_{a}^{1}+i r_{a}^{2}\right)\right|^{2} \\
& \left.-\left(\left|\phi_{a}\right|^{2}+\left|\sigma_{a}\right|^{2}\right)\left(\left|q_{a}\right|^{2}+\left|\widetilde{q}_{a}\right|^{2}+g^{2}\right)\right\}
\end{aligned}
$$

and $\mathcal{L}_{\text {top }}$ is the topological term

$$
\mathcal{L}_{\text {top }}=\sum_{a=1}^{k}\left(\theta-\theta_{a}\right) F_{01}^{a}
$$

Note that due to this axionic coupling the field $\theta$ becomes a compact boson $\theta \sim \theta+2 \pi$. Note also that the total space of parameters $\left\{\left(\vec{r}_{a}, \theta_{a}\right)\right\}_{a=1}^{k}$ is $\left(\mathbf{R}^{3} \times \mathbf{S}^{1}\right)^{k} / S_{k}$. The quotient by $S_{k}$ comes from the fact that the permutation of the $k$ gauge groups leads to the same theory. We will see in section 2.3 that this parameter space has the spacetime interpretation as the moduli space of $k$ H-monopoles.

\subsection{The Low Energy Theory}

Now let us consider the effective theory of this model by restricting the Lagrangian to the massless modes. From the expression of $\mathcal{L}_{\text {pot }}(2.6)$, the moduli space of vacua 2 is characterized by the equations

$$
F_{01}^{a}=\sigma_{a}=\phi_{a}=0, \quad\left|q_{a}\right|^{2}-\left|\widetilde{q}_{a}\right|^{2}=r^{3}-r_{a}^{3}, \quad 2 q_{a} \widetilde{q}_{a}=r^{1}+i r^{2}-\left(r_{a}^{1}+i r_{a}^{2}\right) .
$$

2 Strictly speaking, there is no moduli space of vacua in two dimensions because of the Coleman theorem [14]. We analyze the low energy theory in the spirit of Born-Oppenheimer approximation. 
From the last two equations in (2.8), it follows that

$$
\left|q_{a}\right|^{2}+\left|\widetilde{q}_{a}\right|^{2}=\left|\vec{r}-\vec{r}_{a}\right|
$$

In the IR limit $e_{a} \rightarrow \infty$, the vector multiplet and charged hypermultiplets become massive and they can be integrated out. The crucial step is to rewrite the kinetic term of hypermultiplet restricted on the locus (2.8)

$$
\left|\mathcal{D} q_{a}\right|^{2}+\left|\mathcal{D} \widetilde{q}_{a}\right|^{2}=\frac{1}{4\left|\vec{r}-\vec{r}_{a}\right|}(\partial \vec{r})^{2}+\frac{\left|\vec{r}-\vec{r}_{a}\right|}{4}\left(2 A^{a}+2 \partial \varphi_{a}+\vec{\omega}_{a} \cdot \partial \vec{r}\right)^{2}
$$

where $\varphi_{a}=-\arg \left(i q_{a}\right)$ and $\vec{\omega}_{a}$ is given by

$$
\nabla \times \vec{\omega}_{a}=\nabla \frac{1}{\left|\vec{r}-\vec{r}_{a}\right|}
$$

After setting $\frac{1}{2 e_{a}^{2}}\left(F_{01}^{a}\right)^{2} \rightarrow 0$, the action becomes quadratic in $A_{\mu}^{a}$. Therefore we can integrate out the gauge field classically and we find

$$
A_{\mu}^{a}=-\partial_{\mu} \varphi_{a}-\frac{1}{2} \vec{\omega}_{a} \cdot \partial_{\mu} \vec{r}+\frac{1}{2\left|\vec{r}-\vec{r}_{a}\right|} \epsilon_{\mu \nu} \partial^{\nu} \theta
$$

Plugging this back into the original Lagrangian, the effective Lagrangian for the twisted hypermultiplet is found to be

$$
\mathcal{L}_{\text {eff }}=\frac{1}{2} H\left(\partial_{\mu} \vec{r} \cdot \partial^{\mu} \vec{r}+\partial_{\mu} \theta \partial^{\mu} \theta\right)+\epsilon_{\mu \nu} \vec{\omega} \cdot \partial^{\mu} \vec{r} \partial^{\nu} \theta
$$

where

$$
H=\frac{1}{g^{2}}+\frac{1}{2} \sum_{a=1}^{k} \frac{1}{\left|\vec{r}-\vec{r}_{a}\right|}, \quad \vec{\omega}=\frac{1}{2} \sum_{a=1}^{k} \vec{\omega}_{a}, \quad \nabla \times \vec{\omega}=\nabla H .
$$

This Lagrangian (2.13) is nothing but the nonlinear sigma model describing the $k$ NS5branes smeared in the $\mathbf{S}_{\theta}^{1}$ direction. The last term in 2.13) represents the coupling to the B-field generated by the NS5-branes. As discussed in [13], the localization in the $\theta$ direction is recovered by summing over the instanton effects, which we will turn next.

\subsection{Worldsheet Instanton Corrections}

The instanton effects in the $k=1$ model is studied in [13. In the case of $k \mathrm{H}$ monopoles, we expect that the instanton effects in different $U(1)$ sectors are decoupled from each other in the first approximation. Therefore, we can simply use the result of [13] 
for the single $U(1)$ theory and sum over the $k U(1)$ contributions. The instanton action in the $a^{\text {th }} U(1)$ sector is given by 13

$$
S_{n_{a}}^{\text {inst }}=\left|n_{a}\right|\left|\vec{r}-\overrightarrow{r_{a}}\right|-i n_{a}\left(\theta-\theta_{a}\right)
$$

where $n_{a}$ is the instanton number in the $a^{\text {th }} U(1)$ gauge field

$$
n_{a}=-\int_{\Sigma} \frac{F^{a}}{2 \pi} .
$$

In general it is quite difficult to compute the $n$-instanton effect since it involves a complicated integral over the instanton moduli space. However, it is conjectured that all instanton numbers contribute with equal weight in the effective metric [13] to match the target space picture. Applying this conjecture to our case, we expect that $H$ in (2.14) is corrected to

$$
\begin{aligned}
H & =\frac{1}{g^{2}}+\frac{1}{2} \sum_{a=1}^{k} \frac{1}{\left|\vec{r}-\overrightarrow{r_{a}}\right|} \sum_{n_{a} \in \mathbb{Z}} e^{-\left|n_{a}\right|\left|\vec{r}-\overrightarrow{r_{a}}\right|+i n_{a}\left(\theta-\theta_{a}\right)} \\
& =\frac{1}{g^{2}}+\frac{1}{2} \sum_{a=1}^{k} \frac{\sinh \left|\vec{r}-\overrightarrow{r_{a}}\right|}{\left|\vec{r}-\overrightarrow{r_{a}}\right|} \frac{1}{\cosh \left|\vec{r}-\overrightarrow{r_{a}}\right|-\cos \left(\theta-\theta_{a}\right)} \\
& =\frac{1}{g^{2}}+\sum_{a=1}^{k} \sum_{m_{a} \in \mathbb{Z}} \frac{1}{\left|\vec{r}-\overrightarrow{r_{a}}\right|^{2}+\left(\theta-\theta_{a}+2 \pi m_{a}\right)^{2}} .
\end{aligned}
$$

The sigma model (2.13) with this instanton corrected $H(2.17)$ corresponds to the $k$ NS5branes localized at $\left(\vec{r}_{a}, \theta_{a}\right) \in \mathbf{R}^{3} \times \mathbf{S}^{1}$. From the last expression in (2.17), we can see that this corresponds to the periodic array of CHS solution [15]. This effective theory preserves a $\mathcal{N}=(4,4)$ SUSY since it is a nonlinear sigma model of hyperKähler with torsion [16, 177. As is clear from the derivation, the collective coordinates $\left(\vec{r}_{a}, \theta_{a}\right)$ of NS5-branes appear in the GLSM as the FI-parameter and the theta-parameter.

From the first expression of $H$ in (2.17), we can see that the instanton number $n_{a}$ has a spacetime interpretation as the momentum along $\mathbf{S}_{\theta}^{1}$ and the collective coordinates $\theta_{a}$ 's are conjugate to this momentum mode. In other words, $\theta_{a}$ is the translation mode in the $\mathbf{S}_{\theta}^{1}$ direction. 


\section{GLSM for KK-monopoles}

In this section, we consider the T-dual of H-monopole GLSM (2.1) following the general prescription [18]. We dualize the $\mathcal{N}=2$ twisted chiral multiplet $\Theta$ as was done for the $k=1$ case [11]. To do this, we first rewrite the $F$-term involving $\Theta$ as a $D$-term

$$
\int d^{4} \theta-\frac{1}{g^{2}} \Theta^{\dagger} \Theta-\left(\Theta+\Theta^{\dagger}\right) \sum_{a=1}^{k} V_{a} .
$$

Then we use the usual trick of introducing a real superfield $B$ and a chiral superfield $P$

$$
\int d^{4} \theta-\frac{1}{2 g^{2}} B^{2}-\frac{1}{g^{2}}\left(P+P^{\dagger}+g^{2} \sum_{a=1}^{k} V_{a}\right) B
$$

(3.2) goes back to (3.1) by integrating out $P$, while integrating out $B$ leads to

$$
\int d^{4} \theta \frac{1}{2 g^{2}}\left(P+P^{\dagger}+g^{2} \sum_{a=1}^{k} V_{a}\right)^{2} .
$$

Therefore, the T-dual of (2.1) is given by

$$
\begin{aligned}
\mathcal{L}_{D}= & \int d^{4} \theta \frac{1}{g^{2}} \Psi^{\dagger} \Psi+\frac{1}{2 g^{2}}\left(P+P^{\dagger}+g^{2} \sum_{a=1}^{k} V_{a}\right)^{2} \\
& +\sum_{a=1}^{k}\left\{\frac{1}{e_{a}^{2}}\left(-\Sigma_{a}^{\dagger} \Sigma_{a}+\Phi_{a}^{\dagger} \Phi_{a}\right)+Q_{a}^{\dagger} e^{V_{a}} Q_{a}+\widetilde{Q}_{a}^{\dagger} e^{-V_{a}} \widetilde{Q}_{a}\right\} \\
\mathcal{L}_{F}= & \int d \theta^{+} d \theta^{-} \sum_{a=1}^{k}\left\{\widetilde{Q}_{a} \Phi_{a} Q_{a}+\left(s_{a}-\Psi\right) \Phi_{a}\right\}, \quad \mathcal{L}_{\widetilde{F}}=\int d \theta^{+} d \bar{\theta}^{-} \sum_{a=1}^{k} t_{a} \Sigma_{a} .
\end{aligned}
$$

Again it is easy to write down the Lagrangian in the component fields. The bosonic part of the Lagrangian reads

$$
\begin{aligned}
\mathcal{L}_{\text {kin }}= & -\frac{1}{2 g^{2}}(\partial \vec{r})^{2}+\frac{g^{2}}{2}\left(\partial \gamma+\sum_{a=1}^{k} A^{a}\right)^{2} \\
+ & \sum_{a=1}^{k}\left\{\frac{1}{2 e_{a}^{2}}\left(\left(F_{01}^{a}\right)^{2}-\left|\partial \phi_{a}\right|^{2}-\left|\partial \sigma_{a}\right|^{2}\right)-\left|\mathcal{D} q_{a}\right|^{2}-\left|\mathcal{D} \widetilde{q}_{a}\right|^{2}\right\} \\
\mathcal{L}_{\text {pot }}= & \sum_{a=1}^{k}\left\{-\frac{e_{a}^{2}}{2}\left(\left|q_{a}\right|^{2}-\left|\widetilde{q}_{a}\right|^{2}-r^{3}+r_{a}^{3}\right)^{2}-\frac{e_{a}^{2}}{2}\left|2 q_{a} \widetilde{q}_{a}-\left(r^{1}+i r^{2}\right)+\left(r_{a}^{1}+i r_{a}^{2}\right)\right|^{2}\right. \\
& \left.-\left(\left|\phi_{a}\right|^{2}+\left|\sigma_{a}\right|^{2}\right)\left(\left|q_{a}\right|^{2}+\left|\widetilde{q}_{a}\right|^{2}+g^{2}\right)\right\} \\
\mathcal{L}_{\text {top }}= & -\sum_{a=1}^{k} \theta_{a} F_{01}^{a},
\end{aligned}
$$


where we denote the scalar component of $P$ as $r^{3}+i g^{2} \gamma$.

Let us comment on some of the properties of this Lagrangian. One important property of this model is that the scalar field $\gamma$ is shifted by all $k U(1)$ gauge transformations

$$
\delta A_{\mu}^{a}=\partial_{\mu} \alpha_{a}, \quad \delta \gamma=-\alpha_{a} \quad(a=1, \cdots, k)
$$

Combining $\gamma$ with the phase $\varphi_{a}=-\arg \left(i q_{a}\right)$ of the hypermultiplet scalar, we can define the gauge invariant field $\kappa$ as

$$
\kappa=\gamma-\sum_{a=1}^{k} \varphi_{a}
$$

Note that there is no dynamical theta angle $\theta$ in $\mathcal{L}_{\text {top }}$ (3.5) since we have dualized the twisted chiral multiplet $\Theta$. It is also worth mentioning that the potential term $\mathcal{L}_{\text {pot }}$ is exactly the same as the H-monopole model (2.6). Therefore, the equation for the moduli space of vacua is the same as the H-monopole case (2.8).

By looking at the gauge symmetry of this model (3.4), we immediately notice that this system gives the hyperKähler quotient construction of multi-Taub-NUT space [19]3. 3 . We can explicitly write down the low energy theory following the procedure in the previous section. By restricting to the vacuum (2.8) and integrating out the gauge fields, we find that $A^{a}$ is given by

$$
A_{\mu}^{a}=-\frac{1}{2\left|\vec{r}-\vec{r}_{a}\right| H}\left(\partial_{\mu} \kappa-\vec{\omega} \cdot \partial_{\mu} \vec{r}\right)-\partial_{\mu} \varphi_{a}-\frac{1}{2} \vec{\omega}_{a} \cdot \partial_{\mu} \vec{r} .
$$

where $\vec{\omega}_{a}$ and $H, \vec{\omega}$ are defined in (2.11) and (2.14). Plugging this back into the Lagrangian, the low energy effective Lagrangian is given by

$$
\mathcal{L}_{\text {eff }}=\frac{1}{2} H(\partial \vec{r})^{2}+\frac{1}{2 H}(\partial \kappa-\vec{\omega} \cdot \partial \vec{r})^{2} .
$$

This is nothing but the metric of the multi-Taub-NUT space $M_{k}$ 221,22,. As expected from the supergravity analysis of the B-field zero-mode [10], the curvature $F^{a}=d A^{a}$ of the forms (3.8) are equal to the $k$ linearly independent $L^{2}$-normalizable self-dual harmonic two-forms on the multi-Taub-NUT space [23] 1 目.

3 A similar model in three dimensions was considered in [20].

4 We make slight abuse of notation that we identify the forms on the effective target space (i.e. Taub-NUT) and their pull-back to the worldsheet.

5 Note the difference of the $L^{2}$ harmonic two-forms on the ALF space (Taub-NUT) and the ALE space 24]

$$
\operatorname{dim} L^{2} \mathcal{H}^{2}\left(\mathrm{ALF}_{A_{k-1}}\right)=k, \quad \operatorname{dim} L^{2} \mathcal{H}^{2}\left(\mathrm{ALE}_{A_{k-1}}\right)=k-1
$$




\section{The Physical Meaning of the Collective Coordinate $\theta_{a}$}

We have seen in section 2 that the instanton numbers in the H-monopole model correspond to the momentum modes in the target space and the collective coordinate $\theta_{a}$ (or theta-parameter in GLSM) is the translation mode in the $\mathbf{S}_{\theta}^{1}$. From the general property of T-duality, we expect that the instanton number in the KK-monopole model corresponds to the winding number in the target space picture and the conjugate collective coordinate $\theta_{a}$ is the translation mode in the "winding coordinate" $\mathbf{S}_{\tilde{\theta}}^{1}$. The instanton corrections in the KK-monopole model is analyzed in the UV gauge theory language in [11] for the $k=1$ case. In this section we consider the instanton effect from the low energy sigma model perspective.

As mentioned in the introduction, from the effective theory point of view it seems puzzling to have a nontrivial instanton correction for the $k=1$ case since there is no closed two-cycle in that case. Here we propose that the relevant instanton correction is coming from a disk instanton. The possibility of disk instanton is suggested in [7] in relation to the description of KK-monopoles in the string field theory.

Since we are considering the sigma model on the sphere $\Sigma=\mathbf{S}^{2}$, it seems impossible to have a disk instanton. However, because of the non-compactness of the target space we can consider a map which effectively looks like a disk. We decompose the worldsheet sphere into a disk and one point

$$
\mathbf{S}^{2}=D^{2} \cup\{\infty\}
$$

and consider a map which sends the disk into a finite region of target space and the $\infty$ of worldsheet to the infinity of target space. With this understanding, we can talk about the disk instanton.

Let us consider the configuration of disk instanton which wraps $n$ times on the semiinfinite cigar in the target space

$$
D^{2}=\{z ;|z|<1\} \longrightarrow n C_{a}=\left\{\vec{r}(z)=\vec{r}_{a}+f(|z|) \vec{v}, \kappa(z)=n \arg (z)\right\}
$$

where $\vec{v}$ is a constant unit vector specifying the direction of cigar. The function $f(|z|)$ satisfies the boundary condition

$$
f(0)=0, \quad f(1)=\infty .
$$

6 We do not try to define $\widetilde{\theta}$ as a worldsheet field [11]. Rather, the collective coordinates $\theta_{a}$ appear in the worldsheet action as parameters. By construction, the GLSM of H-monopoles and the GLSM of KK-monopoles have the same parameter space $\left(\mathbf{R}^{3} \times \mathbf{S}^{1}\right)^{k} / S_{k}$. 
$f(|z|)$ can be determined by minimizing the action but we don't need its explicit form. We can see that $C_{a}$ has a shape of cigar by noting that $\mathbf{S}^{1}$ fiber is degenerate at $\vec{r}=\vec{r}_{a}$ and the radius of $\mathbf{S}^{1}$ fiber approaches a constant value $g$ at infinity. If we regard $\tau=1 /|z|$ as the Euclidean time coordinate on the worldsheet, the configuration (4.2) represents a process that a string winding $n$ times around the $\mathbf{S}^{1}$ fiber at infinity propagates towards one of the monopole cores $\vec{r}=\vec{r}_{a}$ and unwinds at $\tau=\infty[7]$.

One can compute the flux of the harmonic two-form $F^{a}=d A^{a}$ (3.8) through the cigar $C_{a}$

$$
\int_{n C_{a}} \frac{F^{b}}{2 \pi}=n \delta_{a, b} .
$$

In this computation only the first term in (3.8) contributes. Other terms in (3.8) couple to the "unwinding" string away from the core of monopoles [7]. We can understand the diagonal property (4.4) in a more abstract way. It is known that the intersection form of the harmonic two-forms $F^{a}=d A^{a}$ given by (3.8) is diagonal [23]

$$
\int_{M_{k}} \frac{F^{a}}{2 \pi} \wedge \frac{F^{b}}{2 \pi}=\delta_{a, b}
$$

Therefor we can define $C_{a}$ as a Poincare dual of the $F^{a}$

$$
C_{a}=P D\left(\left[\frac{F^{a}}{2 \pi}\right]\right) .
$$

From this discussion it is clear that the instanton number in the low energy picture is the winding number of disk instanton wrapped around the cigar. The instanton action in the UV gauge theory picture is obtained in [11]

$$
S^{\text {inst }}=|n|\left|\vec{r}-\vec{r}_{a}\right|-i n \theta_{a}
$$

We do not try to reproduce this instanton action from the low energy point of view. However, we note that the relevant instanton in the UV computation is the constrained instanton defined in the $g \rightarrow 0$ limit [13,11]. In this limit the asymptotic radius $g$ of the $\mathbf{S}^{1}$ fiber goes to zero. Therefore it is possible that the instaton action calculated in the low energy theory is finite despite the fact that the cigar $C_{a}$ is non-compact. In general the low energy theory does not necessarily give the same instanton action as the UV theory. However we expect that the topological charge should have the same interpretation in the UV and IR. What we have shown is that the disk instanton wrapped around $C_{a}$ has the same topological charge as the gauge theory instanton of $a^{\text {th }} U(1)$. 
When $k>1$ one can also consider a sphere instanton which wraps the closed two-cycle in the Taub-NUT

$$
\Sigma=\mathbf{S}^{2} \longrightarrow C_{a}-C_{b}
$$

When the two KK-monopoles coincide $\vec{r}_{a}=\vec{r}_{b}$, the corresponding cycle $C_{a}-C_{b}$ vanishes. From the duality to H-monopole, we expect that the coincident KK-monopoles would show the throat behavior. It would be interesting to see this explicitly using the GLSM. It would be also interesting to determine the precise form of the instanton correction to the KKmonopole sigma model. It is observed [11] that not only the metric is corrected but also the torsion is generated. Parhaps it might be useful to consider the topological A-twist of the KK-monopole GLSM. It would be also interesting to construct a GLSM of the $D_{k}$-type Taub-NUT space [25] and a $\mathcal{N}=(4,0)$ GLSM describing a bound state of H-monopoles and KK-monopoles.

Acknowledgment: I would like to thank Kazuyuki Furuuchi for encouragement. I would also like to thank Steuard Jensen and David Tong for correspondence. 


\section{References}

[1] T. Banks, M. Dine, H. Dykstra and W. Fischler, "Magnetic Monopole Solutions Of String Theory," Phys. Lett. B 212, 45 (1988).

[2] J. P. Gauntlett, J. A. Harvey and J. T. Liu, "Magnetic monopoles in string theory," Nucl. Phys. B 409, 363 (1993) arXiv:hep-th/9211056.

[3] R. R. Khuri, "A Multimonopole solution in string theory," Phys. Lett. B 294, 325 (1992) arXiv:hep-th/9205051.

[4] R. d. Sorkin, "Kaluza-Klein Monopole," Phys. Rev. Lett. 51, 87 (1983).

[5] D. J. Gross and M. J. Perry, "Magnetic Monopoles In Kaluza-Klein Theories," Nucl. Phys. B 226, 29 (1983).

[6] H. Ooguri and C. Vafa, "Two-Dimensional Black Hole and Singularities of CY Manifolds," Nucl. Phys. B 463, 55 (1996) [arXiv:hep-th/9511164].

[7] R. Gregory, J. A. Harvey and G. W. Moore, "Unwinding strings and T-duality of Kaluza-Klein and H-monopoles," Adv. Theor. Math. Phys. 1, 283 (1997) arXiv:hepth/9708086].

[8] N. Seiberg, "New theories in six dimensions and matrix description of M-theory on $\mathrm{T}^{* *} 5$ and $\mathrm{T}^{* *} 5 / \mathrm{Z}(2)$," Phys. Lett. B 408, 98 (1997) arXiv:hep-th/9705221.

[9] N. Seiberg and S. Sethi, "Comments on Neveu-Schwarz five-branes," Adv. Theor. Math. Phys. 1, 259 (1998) arXiv:hep-th/9708085].

[10] A. Sen, "Kaluza-Klein dyons in string theory," Phys. Rev. Lett. 79, 1619 (1997) arXiv:hep-th/9705212.

[11] J. A. Harvey and S. Jensen, "Worldsheet instanton corrections to the Kaluza-Klein monopole," arXiv:hep-th/0507204.

[12] E. Witten, "Phases of $\mathrm{N}=2$ theories in two dimensions," Nucl. Phys. B 403, 159 (1993) arXiv:hep-th/9301042.

[13] D. Tong, "NS5-branes, T-duality and worldsheet instantons," JHEP 0207, 013 (2002) arXiv:hep-th/0204186.

[14] S. R. Coleman, "There Are No Goldstone Bosons In Two-Dimensions," Commun. Math. Phys. 31, 259 (1973).

[15] C. G. . Callan, J. A. Harvey and A. Strominger, "Supersymmetric string solitons," arXiv:hep-th/9112030.

[16] S. J. . Gates, C. M. Hull and M. Rocek, "Twisted Multiplets And New Supersymmetric Nonlinear Sigma Models," Nucl. Phys. B 248, 157 (1984).

[17] G. W. Gibbons, G. Papadopoulos and K. S. Stelle, "HKT and OKT geometries on soliton black hole moduli spaces," Nucl. Phys. B 508, 623 (1997) arXiv:hep-th/9706207.

[18] M. Rocek and E. P. Verlinde, "Duality, quotients, and currents," Nucl. Phys. B 373, 630 (1992) arXiv:hep-th/9110053. 
[19] G. W. Gibbons and P. Rychenkova, "HyperKaehler quotient construction of BPS monopole moduli spaces," Commun. Math. Phys. 186, 585 (1997) arXiv:hepth/9608085.

[20] A. Kapustin and M. J. Strassler, "On mirror symmetry in three dimensional Abelian gauge theories," JHEP 9904, 021 (1999) arXiv:hep-th/9902033.

[21] S. W. Hawking, "Gravitational Instantons," Phys. Lett. A 60, 81 (1977).

[22] G. W. Gibbons and S. W. Hawking, "Classification Of Gravitational Instanton Symmetries," Commun. Math. Phys. 66, 291 (1979).

[23] P. J. Ruback, "The Motion Of Kaluza-Klein Monopoles," Commun. Math. Phys. 107, 93 (1986).

[24] T. Hausel, E. Hunsicker and R. Mazzeo, "Hodge cohomology of gravitational instantons," arXiv:math.dg/0207169.

[25] S. A. Cherkis and A. Kapustin, " $\mathrm{D}(\mathrm{k})$ gravitational instantons and Nahm equations," Adv. Theor. Math. Phys. 2, 1287 (1999) [arXiv:hep-th/9803112]; "Singular monopoles and gravitational instantons," Commun. Math. Phys. 203, 713 (1999) arXiv:hepth/9803160|. 